\title{
Avaliação de Pacientes com Epilepsias Parciais Refratárias às Drogas Antiepilépticas
}

\author{
Juliana Starling Lage* \\ Eliana Garzon* \\ Américo Ceiki Sakamoto** \\ Elza Márcia Targas Yacubian***
}

\begin{abstract}
RESUMO
Pacientes com epilepsia parcial refratária às drogas antiepilépticas necessitam de avaliação multidisciplinar (composta por neurofisiologistas, neurorradiologistas, neuropatologistas e neuropsicólogos) para avaliação e delimitação precisa da área epileptogênica, pois estes podem se beneficiar do tratamento cirúrgico. Este artigo discorre sobre os métodos de avaliação disponíveis.
\end{abstract}

\section{UNITERMOS}

Epilepsia refratária, avaliação pré-operatória, vídeo-EEG, neuroimagem, neuropatologia.
* Centro de Epilepsia de São Paulo, Hospital Alemão Oswaldo Cruz.

** Faculdade de Medicina da USP, Ribeirão Preto. Centro de Epilepsia de São Paulo, Hospital Alemão Oswaldo Cruz.

*** Hospital das Clínicas da FMUSP, São Paulo. Centro de Epilepsia de São Paulo, Hospital Alemão Oswaldo Cruz.

\section{NTRODUÇÃO}

Em estudos norte-americanos, a epilepsia tem incidência de 48,7/100.000 e prevalência de 5,7/1.000 habitantes ${ }^{1}$, e, na América do Sul, estima-se uma prevalência duas vezes mais alta, em média. Fernandes et al. ${ }^{2}$ encontraram, em Porto Alegre, prevalência de 16,5 e 20,3/1.000 para epilepsia ativa e inativa, respectivamente.

Aproximadamente $60 \%$ a $80 \%$ dos casos de epilepsia podem ser controlados com drogas antiepilépticas (DAE) ${ }^{3,4}$.

Não há definição exata do que seja epilepsia refratária. Schmidt ${ }^{5}$ reconhece que a intratabilidade não pode ser considerada "um fenômeno tudo ou nada" e propõe uma escala para a intratabilidade. Gilman et al. ${ }^{6}$ consideram refratários os casos resistentes às drogas de primeira escolha para o tratamento das epilepsias, desde que usadas até níveis máximos tolerados, em monoterapia ou em associação. A freqüência de epilepsia refratária na população não é claramente conhecida. A incidência cumulativa de 135/100.000 tem sido computada de um total de 1.024 pacientes com diferentes tipos de epilepsia ${ }^{5}$.

Em relação à intratabilidade, devem-se levar em conta vários fatores como idade e perspectiva do paciente, nível de tolerabilidade em relação às crises, tipo e prognóstico da epilepsia e, ainda, opções terapêuticas alternativas ${ }^{7}$. Por exemplo, se um tratamento cirúrgico satisfatório parece possível, o tipo e a duração das tentativas medicamentosas não devem ser os mesmos daqueles aplicados a pacientes para os quais nenhuma outra opção parece razoável ${ }^{8}$.

\section{NVESTIGAÇÃO DIAGNÓSTICA}

Muitos erros de diagnóstico são conseqüência de excessiva confiança na impressão inicial ou em qualquer outra investigação prévia. É necessária, sempre, uma reavaliação periódica do caso considerado refratário às DAE. 
Neurologistas e epileptologistas são freqüentemente solicitados para decidir quanto ao diagnóstico entre eventos epilépticos e não epilépticos. Em muitas circunstâncias, mesmo para médicos experientes, o diagnóstico diferencial entre as duas condições pode ser extremamente difícil ${ }^{9}$. Fenômenos como síncope, perda de fôlego, movimentos estereotipados, enxaqueca e uma série de outras condições, inclusive o diagnóstico diferencial entre crises epilépticas e pseudo-epilépticas devem ser investigados antes do diagnóstico de epilepsia refratária.

A dosagem dos níveis séricos das DAE utilizadas deve ser rotineiramente realizada, pois casos de nãoadesão ao tratamento podem ser falsamente interpretados como refratariedade.

\section{ESTUDOS NEUROFISIOLÓGICOS}

Diante de um paciente com epilepsia refratária às DAE, o tratamento cirúrgico é uma das alternativas terapêuticas.

A importância do EEG, especialmente do EEG ictal, tem sido muito enfatizada ${ }^{10}$. O EEG pode ser utilizado de maneira muito mais produtiva com os equipamentos modernos e, principalmente, quando associado ao vídeo, podendo confirmar ou afastar o diagnóstico clínico de epilepsia, identificar os tipos de crises e das síndromes epilépticas, quantificar objetivamente cada um dos eventos, assim como demonstrar se determinada lesão estrutural é ou não epileptogênica ${ }^{11}$. $\mathrm{O}$ sistema 10-20 para a colocação de eletrodos é suficiente para o registro de rotina, mas, em situações especiais, principalmente em técnicas de monitorização por vídeo-EEG, há necessidade do uso de eletrodos adicionais ou especiais, como do esfenoidal e do supra-orbitário, indicados para avaliação das regiões temporobasal e orbitofrontal.

O principal objetivo da avaliação pré-cirúrgica é a identificação da zona epileptogênica, definida como a área do córtex necessária e suficiente para iniciar as crises epilépticas, sendo sua remoção (ou desconexão) fundamental para o controle completo das crises ${ }^{12}$. A zona de início ictal (área do córtex onde as crises são geradas), incluindo a área de propagação precoce, é parte da zona epileptogênica.

Para pacientes com crises epilépticas refratárias às DAE, a primeira etapa da avaliação é o registro videoeletrencefalográfico. Em seguida, ou paralelamente a ele, uma seqüência de informações deve ser obtida com exames de neuroimagem que incluem ressonância magnética (RM), estudos de tomografia por emissão de fóton único (SPECT), realizados nos períodos ictal e interictal, tomografia por emissão de pósitrons (PET) e avaliação neuropsicológica, incluindo teste de Wada, para que possam ser identificados os candidatos à cirurgia de epilepsia.

Tanto as informações clínicas, quanto as eletrofisiológicas, obtidas pela análise do estudo por vídeoEEG, são importantes quando avaliamos as epilepsias parciais refratárias (Figura 1).

Há situações em que o registro eletrencefalográfico, obtido por meio da avaliação não invasiva, é insuficiente para a localização precisa da zona epileptogênica. Um exemplo disso são as crises intratáveis com extensas zonas epileptogênicas, como nos distúrbios do desenvolvimento cortical ${ }^{13,14}$ (Figura 2). Nestes, nem sempre a zona de início ictal respeita lobos e giros, podendo envolver áreas não contíguas

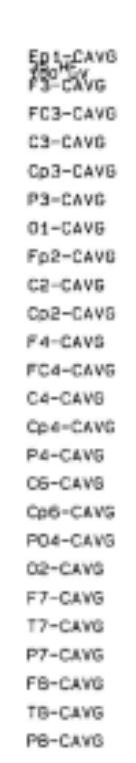

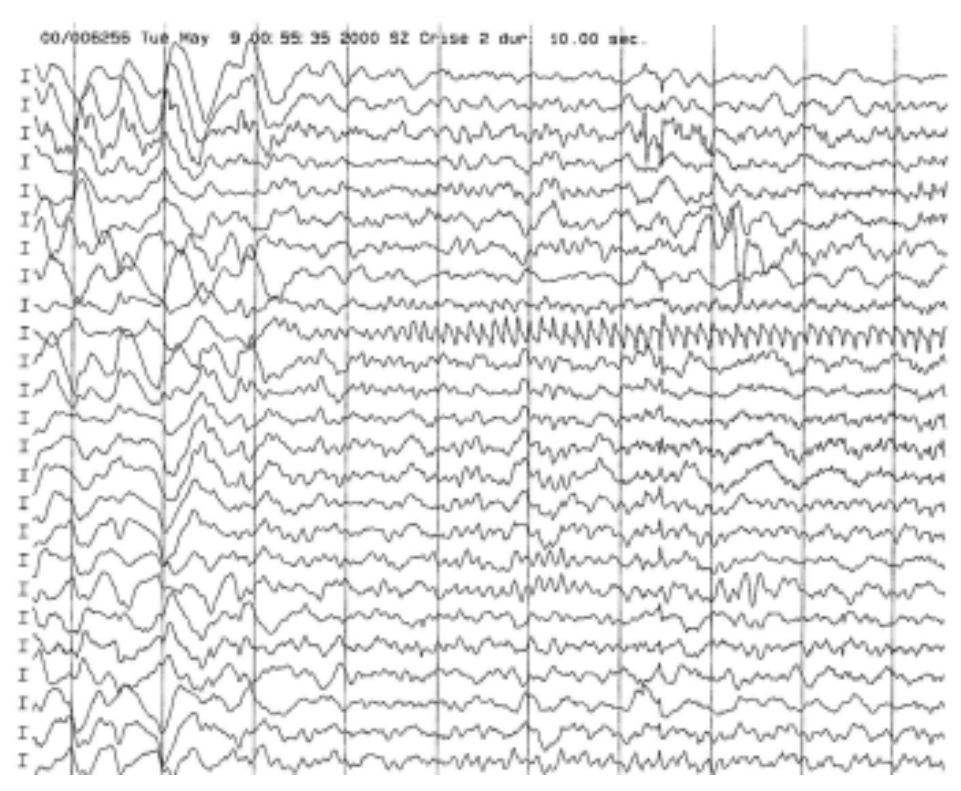

\section{FIGURA 1}

Paciente de 2 anos e 6 meses, com crises refratárias ao tratamento clínico. No estudo de vídeo-EEG, foi verificado que o fenômeno inicial, percebido pela análise da semiologia crítica, deve ter sido sensitivo, pois embora a criança não tenha sido capaz de informar, observou-se, pelo vídeo, que o paciente colocava sua mão no membro inferior esquerdo e, poucos segundos após, foram observadas clonias nesse membro. Observe a descarga ictal no EEG do escalpo restrita ao eletrodo $\mathrm{Cp} 2$. 


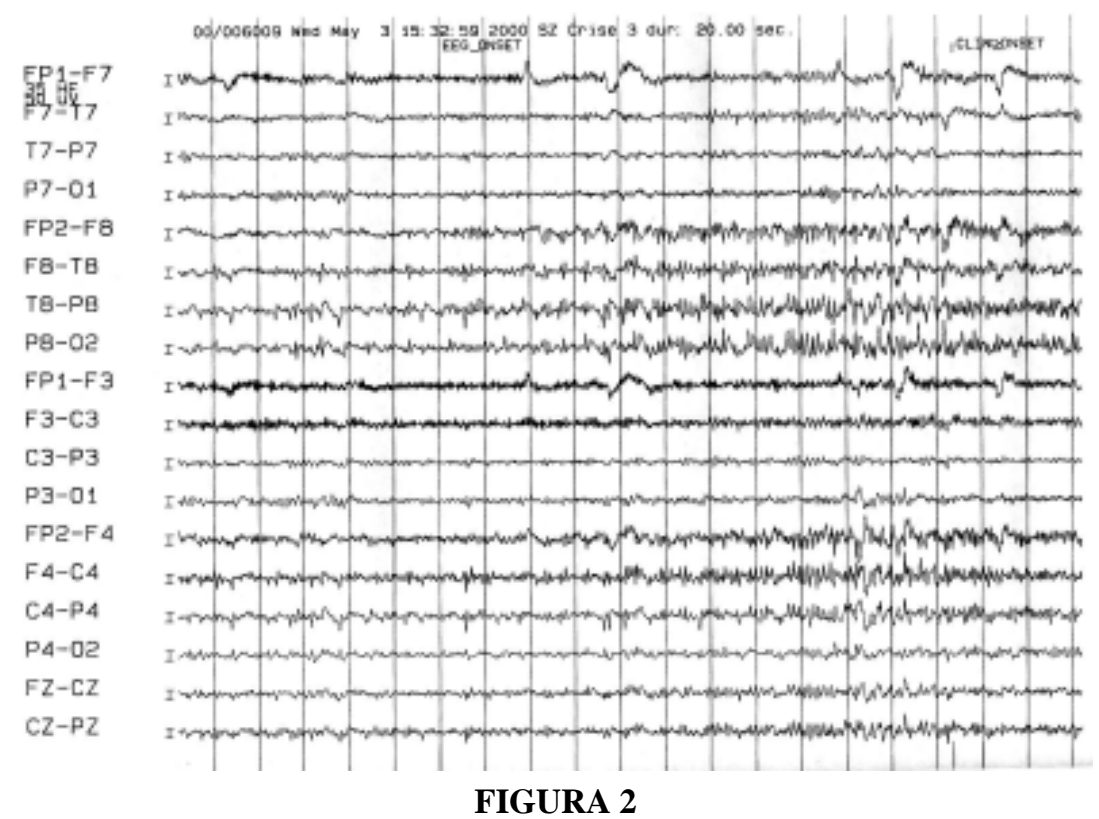

Paciente de 16 anos com crises refratárias secundárias à displasia cortical parieto-occipital direita. Observe que o EEG apenas regionaliza a zona de início ictal. encaminhado para tratamento cirúrgico, mas, ao contrário, se existirem divergências, a próxima etapa envolverá a utilização de outros métodos diagnósticos na avaliação pré-cirúrgica, inclusive avaliação com eletrodos intracranianos. Esta não deverá ser realizada, a menos que os estudos não invasivos tenham gerado, no mínimo, uma hipótese razoável a respeito da localização da possível zona epileptogênica a ser ressecada ${ }^{17}$.

Os estudos eletrofisiológicos invasivos são usados para lateralizar e localizar a área epileptogênica, ao mesmo tempo em que podem determinar a extensão da anormalidade e, em certas circunstâncias, mapear as áreas corticais eloqüentes, com objetivo de evitar seqüelas com o ato cirúrgico. Os procedimentos mais comuns são a eletrocorticografia (ECoG) intra-operatória, a implantação de eletrodos profundos por estereo$\operatorname{taxia}^{17}$ e a colocação de estrias nos espaços subdural ou epidural ${ }^{18}$. Técnicas alternativas

(Figuras 3A e 3B). Há ainda casos em que o tecido displásico e a zona epileptogênica não são coincidentes ${ }^{15,16}$.

Se os dados de semiologia das crises estiverem de acordo com os dados do EEG de escalpo e com os demais testes da avaliação pré-cirúrgica (RM, teste neuropsicológico, teste do amital sódico), o paciente será para a avaliação semi-invasiva, como eletrodos de forame oval ou eletrodos epidurais, têm sido também utilizadas. Poucos centros de cirurgia para epilepsia ainda realizam avaliação invasiva crônica para a maioria dos $\operatorname{casos}^{17}$. No entanto, deve-se salientar que em $10 \%$ a $50 \%$ dos pacientes, a avaliação não invasiva não permite a demonstração de dados localizatórios ${ }^{19}$.
A

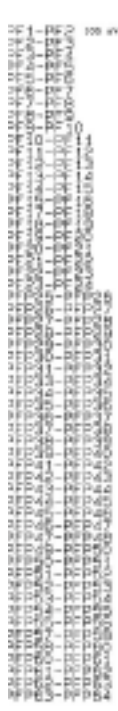

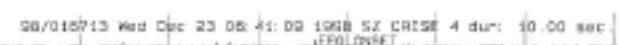

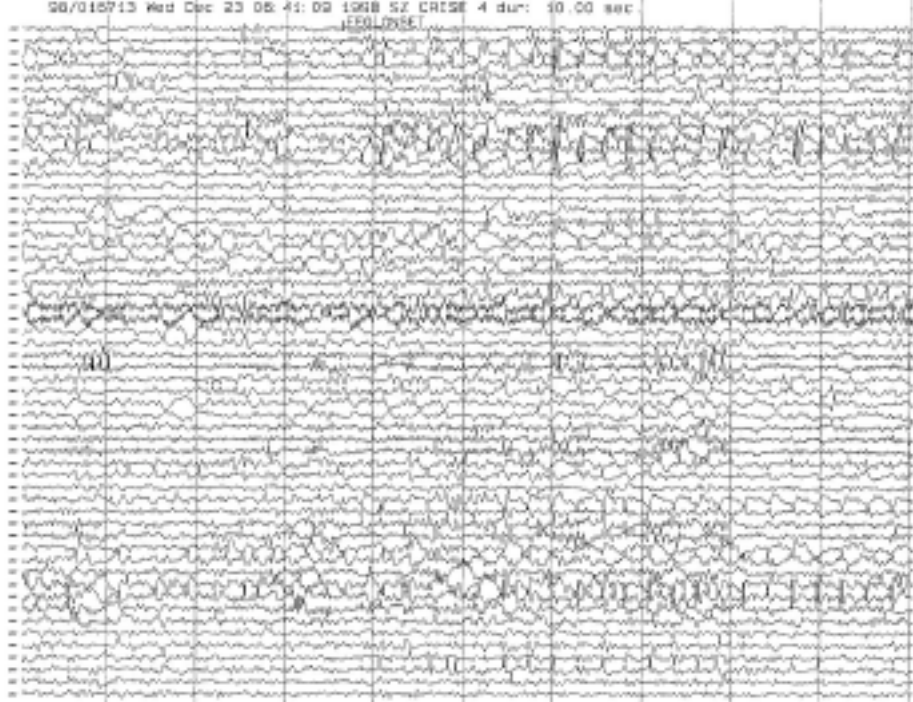

B

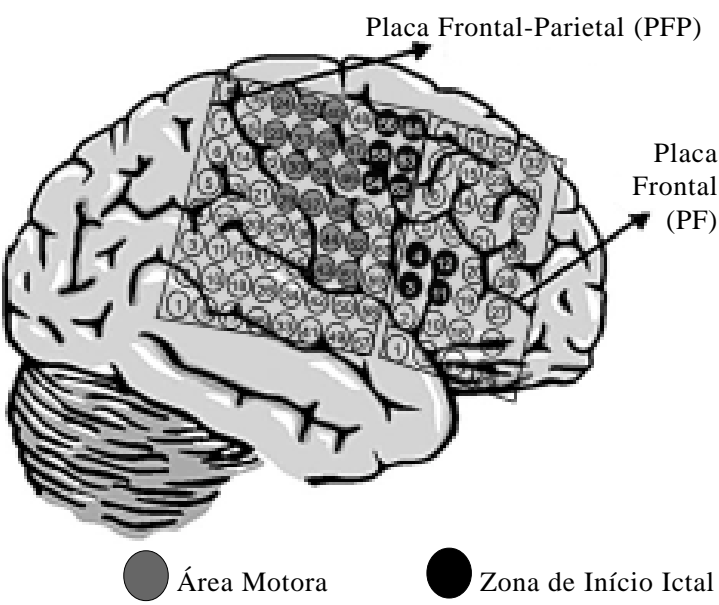

FIGURA 3

A. EEG de paciente de 19 anos, com crises refratárias ao tratamento clínico. Estudo ictal obtido com eletrodos subdurais. Observe a área de início ictal em regiões próximas, mas não contíguas. B. Delimitação da área epileptogênica e sua relação com o córtex eloqüente, após o registro de crises e estimulação cortical. 
Embora alguns serviços ainda prefiram a avaliação da provável zona epileptogênica com registro ECoG intraoperatório $^{20}$, o qual, na verdade, permite apenas a delimitação da zona irritativa, outros utilizam rotineiramente a implantação de eletrodos subdurais ou profundos ${ }^{21}$, que permitirão a caracterização da zona de início ictal e a estimulação de áreas corticais eloqüentes.

Existe porém uma tendência à diminuição da necessidade de avaliação invasiva, à medida que outras técnicas vão fazendo parte da avaliação pré-cirúrgica ${ }^{22}$, particularmente as representadas por testes funcionais, como PET e RM funcional ${ }^{23}$.

\section{NE UROI MAGE M}

O progresso das técnicas de imagem, nas duas últimas décadas, revolucionou a prática da neurologia e da neurocirurgia e, especialmente, a caracterização de lesões discretas relacionadas às epilepsias, pois a capacidade de diferenciar os limites entre substância branca e cinzenta com a RM foi de extrema importância para o diagnóstico dos distúrbios do desenvolvimento cortical, incluindo lesões sutis que correspondem a cerca de $30 \%$ das lesões estruturais de pacientes com epilepsias refratárias (Figura 4).

Os métodos de neuroimagem funcional como os estudos de SPECT, especialmente aqueles realizados no período crítico (Figura 5) e os estudos de PET,

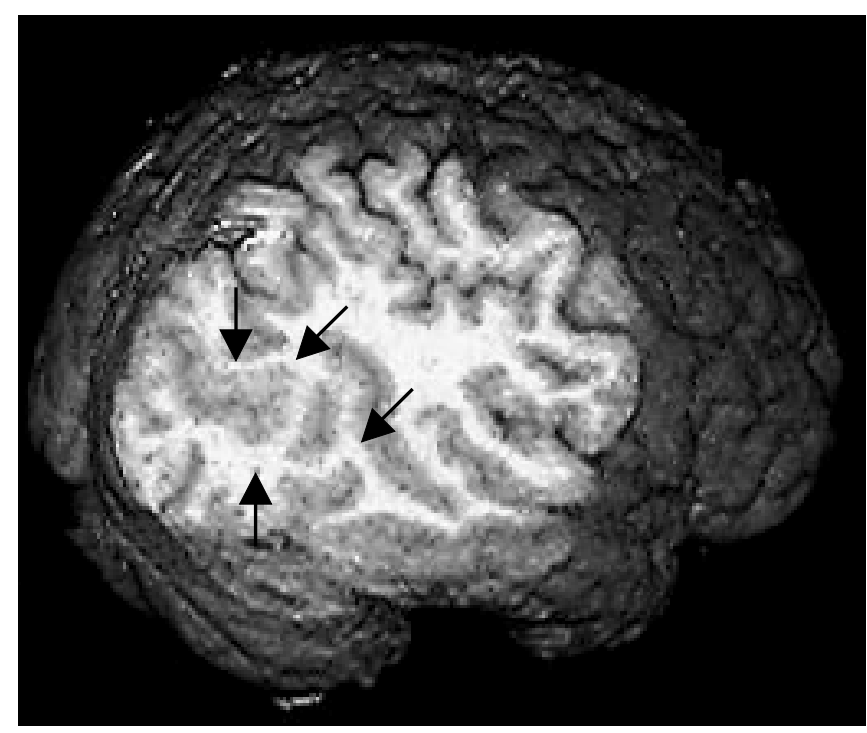

FIGURA 4

Imagem da RM em T1, com reconstrução volumétrica tridimensional, mostrando espessamento giral e perda da diferenciação entre substância branca e cinzenta, na região parietooccipital direita. (Paciente cujo EEG é mostrado na figura 2).

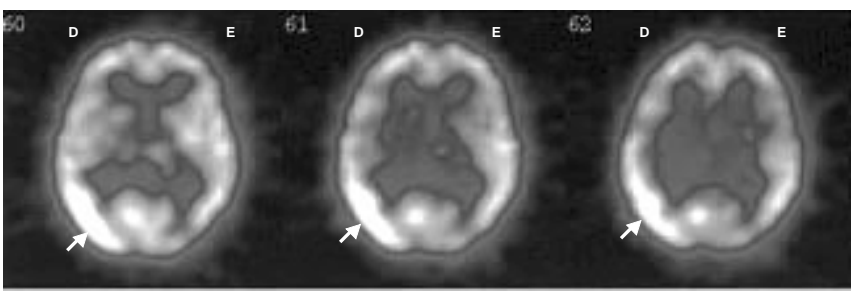

FIGURA 5

Estudo com SPECT ictal realizado na paciente cuja RM é mostrada na figura anterior. Observe a área de aumento acentuado de fluxo sanguiíneo cerebral na região posterior à direita.

permitem o acesso à fisiologia cerebral, pela avaliação do fluxo sangüíneo e da taxa de metabolismo cerebral, respectivamente. Estudos interictais, em pacientes com epilepsias focais, demonstram uma ou mais regiões de hipometabolismo em $75 \%$ a $90 \%{ }^{24}$ e hipoperfusão regional em $50 \%$ a $75 \%$ dos pacientes ${ }^{25}$. Neles, a extensão da área de hipometabolismo ou hipofluxo geralmente é maior que a área envolvida pela atividade epileptogênica avaliada pelo EEG. A importância do estudo funcional é tal que, se um SPECT interictal de um paciente com suposta epilepsia generalizada primária revelar consistentemente uma área de hipoperfusão focal, deve ser considerada a hipótese de epilepsia focal com generalização secundária e, assim, a estratégia terapêutica deverá ser reavaliada ${ }^{26}$. Desde a década de 30 , com os estudos de Penfield ${ }^{27}$, já se sabe que há aumento focal do fluxo sangüíneo cerebral durante crises epilépticas parciais. Explorando esse fenômeno, o estudo de SPECT ictal tem papel importante na avaliação pré-cirúrgica das epilepsias parciais, embora nas epilepsias extratemporais o tempo de hiperperfusão cerebral seja menor que nas crises do lobo temporal. Alguns grupos, como o australiano de Berkovic et al. ${ }^{28}$, têm conseguido localização correta da zona epileptogênica em até $90 \%$ dos casos. Em pacientes com epilepsia extratemporal, em que o EEG e a RM são inconclusivos, o estudo de SPECT ictal pode guiar o planejamento da avaliação invasiva.

Mais recentemente, na avaliação das epilepsias parciais têm sido utilizadas novas técnicas de RM, como a espectroscopia, que permite a mensuração da concentração de substâncias como o $\mathrm{N}$-acetilaspartato (NAA), um marcador da integridade neuronal, a colina, um marcador da desintegração da mielina, o lactato, que se apresenta elevado em processos de sofrimento neuronal, como na fase pós-ictal, e outros marcadores de atividade neuronal, definindo regiões patológicas em áreas restritas. A volumetria por RM possibilita um diagnóstico mais preciso de atrofias discretas, principalmente no estudo das estruturas 
temporais mediais. Em um estudo comparativo realizado em pacientes com epilepsia temporal, a espectroscopia lateralizou corretamente $86 \%$ dos casos, a volumetria, $83 \%$ e a combinação das duas técnicas, $90 \%$ dos casos, contra $93 \%$ de lateralização pelo $E \mathrm{EG}^{29}$. Reduções do NAA são bem correlacionadas com o grau de perda neuronal no estudo histopatológico, mas também podem ocorrer apenas por disfunção neuronal.

Outras técnicas de obtenção e reconstrução das imagens por RM têm evoluído rapidamente nos últimos anos. A RM funcional permite, pela aplicação de estímulos específicos, o mapeamento não invasivo do córtex cerebral. A técnica de reconstrução curvilinear de RM, que combina as características da reconstrução tridimensional com a resolução dos cortes seriados e de cortes curvilíneos que seguem o contorno da convexidade cerebral, permite o reconhecimento de lesões sutis, situadas, por exemplo, na profundidade de um sulco. Foram ainda desenvolvidos programas que permitem a sobreposição das imagens da RM com as obtidas dos estudos de PET e SPECT, facilitando o planejamento cirúrgico e o diagnóstico da área de início ictal.

\section{AVALIAÇÃO NEUROPSICOLÓGICA}

$\mathrm{Na}$ avaliação dos candidatos à cirurgia de epilepsia, os objetivos dos testes neuropsicológicos são avaliar de forma quantitativa as funções cognitivas, estabelecendo uma linha de base para comparações futuras e analisar qualitativamente as funções cognitivas, com a finalidade de localizar disfunções cerebrais regionais. Nas epilepsias temporais, a utilização de testes para avaliação da memória é considerada fundamental, devendo-se levar em conta a diferença funcional que existe entre os lobos temporais, ou seja, o dominante mediando a memória para materiais verbais e o não-dominante, a memória para materiais não verbais ${ }^{30}$.

Em casos com indícios de representação atípica da linguagem, disfunção bitemporal e dificuldades na localização da área epileptogênica, o teste do amital sódico (teste de Wada) pode fornecer informações decisivas para assegurar que a retirada das estruturas mediais temporais não ocasionará novos déficits nas funções de linguagem ou de memória. O lado da disfunção mnésica, detectado pelo teste do amital, geralmente tem boa correlação com os dados de RM do hipocampo e com o grau de perda celular observada nos estudos anatomopatológicos ${ }^{31}$.

\section{ESTUDOS NEUROPATOLÓGICOS}

Os estudos neuropatológicos em epilepsia exigem, hoje, a associação entre laboratórios de neurociências e a equipe multidisciplinar responsável pela avaliação do paciente e buscam respostas a duas questões fundamentais: existe relação causal entre a alteração estrutural encontrada e a ocorrência de crises? Existe um substrato comum a todas as epilepsias?

Apesar da diversidade das lesões potencialmente epileptogênicas (como displasias corticais, tumores e áreas glióticas), o neuropatologista conta com técnicas sofisticadas de imunoistoquímica, microscopia eletrônica, biologia molecular e, mais recentemente, com estudo combinado de microfisiologia e anatomia de tecido ressecado de pacientes portadores de epilepsia intratável. Tal técnica permite o registro eletrofisiológico de uma única célula, fornecendo informações relativas ao potencial de membrana, à configuração dos canais iônicos e da resposta à infusão de DAE. Após essa etapa, as células podem ser coradas, permitindo correlação morfológica e funcional dos vários tipos neuronais encontrados no tecido retirado da suposta área epileptogênica. Esses estudos vão ampliar nossos conhecimentos sobre a fisiopatologia da hiperexcitabilidade da membrana neuronal.

No campo das displasias corticais, os estudos com modelos animais sugerem que esses distúrbios do desenvolvimento cortical possam tanto causar hiperexcitabilidade focal (por reorganização da rede neuronal em torno da área displásica), como difusa (por alteração das propriedades de disparo da célula e formação de pontes entre estruturas normalmente não conectadas) ${ }^{32}$.

Outra proposta de investigação recente é a combinação do registro patch-clamp com amplificação do RNAm, também de célula única. Essa técnica tem permitido a demonstração de alteração na expressão de subunidades do receptor gabaérgico de células granulares do giro denteado da formação hipocampal de pacientes epilépticos ${ }^{33}$.

\section{SUMMARY \\ Evaluation of Patients with Refractory Partial Epilepsy to Drugs The diagnostic and treatment of epilepsy has grown enormously in the last decade thank to explosion of basic information from laboratory neuroscience combined with advances in diagnostic tools, therapeutic approaches and surgical techniques. This article describes the methods used in the evaluation by a muldisciplinary team including neurophysiologists, neuroradiologists, neuropathologists and neuropsychologists.}

\section{KEYWORDS}

Refractory epilepsy, pre-surgery evaluation, video-EEG, neuroimaging, neuropathology. 


\section{Referências}

1. Hauser WA, Kurland LT. The epidemiology of epilepsy in Rochester, Minnesota, 1935 through 1967. Epilepsia, 16:166, 1975

2. Fernandes JG, Schmidt MI, Monte TL, Tozzi S, Sander JWAS. Prevalence of epilepsy: the Porto Alegre study. Epilepsia, 33 (suppl. 3): 132, 1992.

3. Hauser WA, Annegers JF, Kurland LT. Prevalence of epilepsy in Rochester, Minnesota:1940-1980. Epilepsia, 32:429-45, 1991.

4. Hauser W, Hesdorffer DC. The natural history of seizures. In: Wyllie E. (ed.). The treatment of epilepsy: principles and practice, $2^{\text {nd. }}$ ed. Baltimore, Williams \& Wilkins, 1996, 173-8.

5. Schmidt D. Medical intractability in partial epilepsies. In: Lüders H (ed.). Epilepsy surgery. New York, NY, Raven Press, 1991, 83-90.

6. Gilman JT, Duchowny M, Jayakar P, Resnick TH. Medical intratactability in children evaluated for epilepsy surgery. Neurology, 44:1341-3, 1994.

7. Bourgeois BFD. General concepts of medical intractability. In: Lüders H (ed.). Epilepsy surgery. New York, NY, Raven Press, 1991, 77-81.

8. Aicardi J, Shorvon SD. Intractable epilepsy. In: Engel Jr J, Pedley TA (eds.). Epilepsy: a comprehensive textbook. Philadelphia, Lippincott-Raven Publishers, 1997, 1325-31.

9. Sakamoto AC, Holthausen H, Noachtar S. Diagnosis of pseudoepileptic seizures. In: Wolf $\mathrm{P}$ (ed.). Epileptic seizures and syndromes. London, John Libbey \& Company, 1994, 633-42.

10. Duchowny MS, Shewmon DA, Wyllie E, Andermann F, Mizrahi EM. Special considerations for preoperative evaluation in childhood. In: Engel Jr J. (ed.). Surgical treatment of the epilepsies, $2^{\text {nd. }}$ ed. New York, NY, Raven Press, 1993, 415-27.

11. Kaplan PW, Lesser RP. Noninvasive EEG. In: Wyllie E (ed.). The treatment of epilepsy: principles and practice, $2^{\text {nd. }}$ ed., Baltimore, Willians \& Wilkins, 1996, 976-87.

12. Lüders HO, Engel Jr J, Munari C. General principles. In: Engel Jr J (ed.). Surgical treatment of the epilepsies. New York, NY, Raven Press, 1992, 137-53.

13. Awad IA, Rosenfeld R, Ahl J, Hahn JF, Lüders H. Intractable epilepsy and structural lesions of the brain: mapping, resection strategies, and seizure outcome. Epilepsia, 32:17986, 1991.

14. Palmini A, Andermann F, Olivier A, Tampieri D, Robitaille $Y$, Wright G. Focal neuronal migration disorders and intractable partial epilepsy: study of 30 patients. Ann Neurol, 30:741-9, 1991.

15. Spreafico R, Pasquier B, Minotti L. Immunocytochemical investigation on dysplastic human tissue from epileptic patients. Epilepsy Res, 32:34-48, 1998.

16. Garbelli R, Munari C, De Biasi S. Taylor's cortical dysplasia: a confocal and ultrastructural immunohistochemical study. Brain Pathol, 9:445-61,1999.

17. Engel Jr J, Ojemann GA. The next step. In: Engel Jr J (ed.). Surgical treatment of the epilepsies, $2^{\text {nd. }}$ ed. New York, NY, Raven Press, 1993, 319-29.

18. Wyler AR, Ojemann GA, Lettich E, Ward Jr AA. Subdural strip electrodes for localizing epileptogenic foci. J Neurosurg, 60:1195-200, 1984.

19. Spencer SS, So NK, Engel Jr J, Spencer DD. Depth electrodes. In: Engel Jr J (ed.). Surgical treatment of the epilepsies, $2^{\text {nd. }}$ ed. New York, NY, Raven Press, 1993, 359-76.

20. Palmini A, Gambardella A, Andermann F. Intrinsic epileptogenicity of human dysplasic cortex as suggested by corticography and surgical results. Ann Neurol, 37:476-87, 1995.
21. Munari C, Musolino A, Blond S. Stereo-EEG exploration in patients with intractable epilepsy: topographic relations between a lesion and epileptogenic areas. In: Schmidt D, Morselli PL (eds.). Intractable epilepsy: experimental and clinical aspects. New York, NY, Raven Press, 1986, 129-46.

22. Engel Jr J, Henry TR, Risinger MW, Mazziotta JC, Sutherling WW, Levesque MF, Phelps ME. Presurgical evaluation for partial epilepsy: relative contributions of chronic depthelectrode recordings versus FDG-PET and scalp-sphenoidal ictal EEG. Neurology, 40:1670-7, 1990.

23. Engel J, Kuhl DE, Phelps ME, Crandall PH. Comparative localization of epileptic foci in partial epilepsy by PET and EEG. Ann Neurol,12:529-37,1982.

24. Stefan H, Pawlik G, Böcher-Schwarz HG. Functional and morphological abnormalities in temporal lobe epilepsy: a comparison of interictal and ictal EEG,CT, MRI, SPECT and PET. J Neurol, 234:377-84, 1987.

25. Andersen AR, Rogvi-Hansen B, Dam M. Utility of interictal SPECT of rCBF for focal diagnosis of epileptogenic zone(s). Acta Neurol Scand, 152 (suppl.),129-34, 1994.

26. Newton MR, Berkovic SF, Austin MC, Rowe CC, McKay WJ, Bladin PF. SPECT in the localization of extratemporal and temporal seizure foci. J Neurol Neurosurg Psychiatry, 59:2630, 1995.

27. Penfield W, von Santha K, Cipriani A. Cerebral blod flow during induced epileptiform seizures in animals and man. $J$ Neurophysiol, 2:257-67,1939.

28. Berkovic SF, Andermann F, Olivier A, Ethier R, Melanson D, Robitaille Y, Kuzniecky R, Peters T, Feindel W. Hippocampal sclerosis in temporal lobe epilepsy demonstrated by magnetic ressonance imaging. Ann Neurol, 29:175-82, 1991.

29. Cendes F, Caramanos Z, Andermann F, Dubeau F, Arnold $D L$. Proton magnetic resonance spectroscopy imaging and magnetic resonance imaging volumetry in the lateralization of temporal lobe epilepsy. A series of 100 patients. Ann Neurol, 42: 737-46, 1997.

30. Jones-Gotman M. Psychological evaluation for epilepsy surgery. In: Shorvon S, Dreifuss F, Fish D, Thomas D (eds.). The treatment of epilepsy. London, Blackwell Science, 1996, 621-30.

31. Jones-Gotman M, McMackin D, Cendes F. Performance on intracarotid sodium amobarbital memory tests: relationship to hippocampal atrophy as estimated by volumetric MRI. Epilepsia, 34 (suppl. 6):94, 1993.

32. Chevassus-au-Louis N, Baraban SC, Gaïarsa JL, Ben-Ari Y Cortical malformations and epilepsy: new insights from animals models. Epilepsia, 40(7):811-21,1999.

33. Brooks-Kayal AR, Shumate MD, Jin H, Lin DD, Rikhter TY, Holloway KL, Coulter DA. Human neuronal gammaaminobutyric acid $(A)$ receptors: coordinated subunit mRNA expression and functional correlates in individual dentate granule cells. J Neurosci, 19:8312-8, 1999.

\section{Endereço para correspondência:}

Elza Márcia Targas Yacubian

Escola Paulista de Medicina

Disciplina de Neurologia

Rua Botucatu, 740 - Vila Clementino

CEP 04023-900 - São Paulo, SP

E-mail: yacubian@originet.com.br 